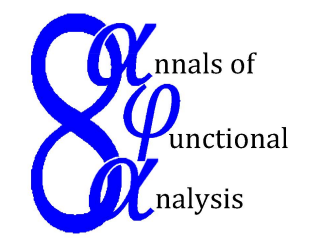

Ann. Funct. Anal. 6 (2015), no. 1, 24-33

http://doi.org/10.15352/afa/06-1-3

ISSN: 2008-8752 (electronic)

http://projecteuclid.org/afa

\title{
COMPLETIONS AND BALLS IN BANACH SPACES
}

\author{
PIER LUIGI PAPINI
}

Communicated by C. P. Niculescu

\begin{abstract}
In this paper we study properties of complete sets and of completions of sets in Banach spaces, with relation to balls: in particular, we study conditions implying that a set is a ball, or it has a completion which is a ball. We also discuss some related simple properties considered in a few recent papers, a new similar property, and relations among classes of sets defined by them. Results are illustrated by several examples.
\end{abstract}

\section{INTRODUCTION}

The notion of diametrically maximal, or complete set, is rather old; a set is complete if all its proper supersets have a larger diameter.

A classical reference for complete sets is the survey paper [8]; for more recent results we indicate [17], [20], and -for finite dimensional spaces- Section 2 in the survey [15].

Recently, classes of sets akin to the class of complete sets have been introduced and studied (see $[17,6])$. Here we continue the study of these classes and of their mutual relationships.

For complete sets, and for sets in some related classes, we can ask which conditions (like symmetry, or relations between radius and diameter) imply that such a set is a ball.

Here we deal with these questions: we single out and discuss some simple properties and a few facts connected with balls.

In Section 2 we study another property for a set, weaker then completeness, and we compare several related conditions. In Section 3 we study conditions

Date: Received: Jan. 9, 2013; Accepted: Mar. 12, 2013.

2010 Mathematics Subject Classification. Primary 46B99; Secondary 52A05, 46B20.

Key words and phrases. Diametrically maximal, ball, constant radius. 
implying that a set is a ball. In Section 4 we deal with sets having a completion that is a ball. Finally, in Section 5, we collect all relevant examples.

Let $X$ be a real Banach space; we denote by $O$ the origin. For $x \in X$ and $r \geq 0$, set

$$
B(x, r)=\{y \in X:\|x-y\| \leq r\} .
$$

Let $D$ be a bounded closed and convex set containing at least two points. In the following we shall always consider sets satisfying this condition. By $\delta(D)$ we will denote the diameter of $D$; by $\partial(D)$ its boundary.

We say that $D$ is complete, or diametrically maximal - (DM) for short - if $\delta(D \cup\{x\})>\delta(D)$ for every $x \notin D$.

A completion of $D$ is a (DM) set $D_{m}$ containing $D$ and such that $\delta(D)=\delta\left(D_{m}\right)$.

Set, for $x \in X$ :

$r(D, x)=\sup \{\|x-d\|: d \in D\}$;

$r(D)=\inf \{r(D, x): x \in X\} \quad$ (radius of $D)$;

$r(D, D)=\inf \{r(D, x): x \in D\} \quad($ self-radius of $D)$.

A point $c \in X$ such that $r(D, c)=r(D)$ is called a center of D. Note that $r(D) \leq r(D, D) \leq \delta(D) \leq 2 r(D)$ always; if $D$ is complete, then $r(D, D)=r(D)$ (see [3, Theorem 3.3]).

Also, let

$r^{\prime}(D, x)=\sup \{\alpha>0: B(x, \alpha) \subset D\}$ for $x$ interior to $D$ and

$r^{\prime}(D, x)=0$ if $x$ is in the boundary of $D$ or outside $D$;

we denote: $r^{\prime}(D)=\sup \left\{r^{\prime}(D, x): x \in D\right\}$.

\section{ANOTHER CONDITION}

We recall that a set $C$ is said to be of constant width - (CW) for short - , equal to $d$, if for every functional $f \in X^{*}$ we have $\sup \{f(x): x \in C\}-\inf \{f(x): x \in$ $C\}=d$; in such case, $d=\delta(D)$.

We say that a set $A$ has constant diameter if it satisfies the following condition: (CD) $r(C, x)=\delta(C)+\operatorname{distance}(x, C)$ for every $x \notin C$.

Balls are sets of constant width, and these in turn are complete. More precisely, we always have:

$$
(\mathrm{CW}) \Rightarrow(\mathrm{CD}) \Rightarrow(\mathrm{DM})
$$

the reverse implications are not true in general: see [17, Proposition 3.2].

The notions of constant width and completeness for a set are equivalent in Minkowskian planes and in Hilbert spaces, see [7]. For results of this type, in finite dimensional spaces, see also [18].

We recall two other conditions, considered in previous papers.

Next condition is satisfied by all (DM) sets (see [6]):

$$
\delta(D)=r(D, D)+r^{\prime}(D) \text {. }
$$


Another condition, that we shall denote by (CR), was studied in [17], where the sets satisfying it were called constant radius sets; then it was considered in [6], where it was denoted by $(3)$ :

(CR) $\quad r(A, x)+r^{\prime}(A, x) \geq \delta(A)$ for all $x \in A$.

Equivalently: $r(A, x)+r^{\prime}(A, x)=\delta(A)$ for all $x \in A$, or also (see [17, pp. 825, $828])$,

$r(A, x)=$ constant $(=\delta(A))$ for every $x$ in $\partial A$.

Condition (CR) lies between completeness and (4); namely (see [6, Section 5]):

$(\mathrm{DM}) \Rightarrow(\mathrm{CR}) \Rightarrow(4)$.

We can consider a new condition which lies between completeness and $(\mathrm{CR})$ : $(\mathrm{CR}+) \quad r(A, x) \geq \delta(A)$ for all $x$ not in the interior of $A$.

Clearly

$(\mathrm{DM}) \Rightarrow(\mathrm{CR}+) \Rightarrow(\mathrm{CR})$.

The converse implications are not true; in fact, Example 5.2 will show that $(\mathrm{CR}+) \nRightarrow(\mathrm{DM})$; Example 5.1 will show that $(\mathrm{CR}) \nRightarrow(\mathrm{CR}+)$.

Consider the following condition (stronger than (4)):

(5) $r(A)+r^{\prime}(A)=\delta(A)$.

More precisely (see [6, Remark 5]), (5) is equivalent to (4) jointly with $r(A)=$ $r(A, A)$.

We are going to show that condition (5) is weaker than $(\mathrm{CR}+)$.

Proposition 2.1. A set satisfying $(C R+)$, also satisfies (5).

Proof. Condition $(\mathrm{CR}+)$ means that $r(A, x)=\delta(A)$ if $x$ is in the boundary of $A$ and $r(A, x) \geq \delta(A)$ if $x \notin A$; so it implies $r(A, A)=r(A)$. Since it is known that (CR) implies (4) (see [6, Lemma 3]), then (CR+) also implies (5); this concludes the proof.

Remark 2.2. Note that the converse of the last result is not true: in fact, as shown in [6, Example 4], (5) $\nRightarrow(\mathrm{CR})$.

Example 5.1 below also shows that $(\mathrm{CR}) \nRightarrow(5)$ (in such example, $r(D) \neq$ $r(D, D))$.

We resume part of the last discussion with the following scheme; the only true implication are those indicated below:

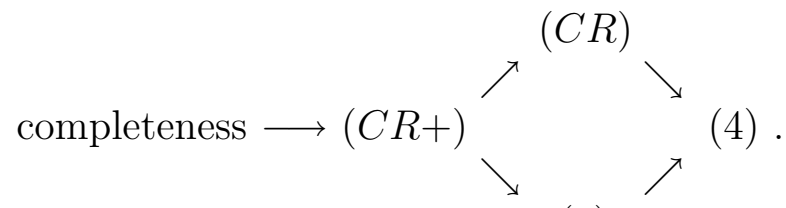

It was indicated in [6] that $(4) \nRightarrow(5)$, but Example 7 in [6] is wrong (for the set $A$ in that example, $\left.r^{\prime}(A)=0\right)$. Indeed $(4) \nRightarrow(5)$ (see Example 5.1 below): 
but refining the proof of [17, Proposition 2.2], we shall prove that this implication is true if $A$ has nonempty interior. So for sets with nonempty interior, we have (for the first part see the cited proposition of [17]):

$$
(\mathrm{DM}) \Longleftrightarrow(\mathrm{CR}+) \Longleftrightarrow(\mathrm{CR}) ; \quad(4) \Longleftrightarrow(5) \text {. }
$$

Note that, in particular, if the space $X$ is finite dimensional, then $r(A, A)<$ $\delta(A)$ always, so already condition (4) implies in this case nonemptiness for the interior of $A$. The same, if $X$ is a Hilbert space.

Proposition 2.3. If A satisfies (4) and its interior is nonempty, then A satisfies (5).

Proof. Let $r^{\prime}(A)>0$. It is not a restriction to assume that $\delta(A)=1$; let $r^{\prime}=$ $r^{\prime}(A)>0 ; r(A, A)+r^{\prime}(A)=\delta(A)=1$. We must prove that $r(A)+r^{\prime}=1$.

Assume that, for some $\sigma>0, r(A)=1-r^{\prime}-\sigma<r(A, A)$. If $r(A)=r^{\prime}$, then $A$ is a ball (see Theorem 3.1 below), and then $r(A)+r^{\prime}(A)=\delta(A)$. Otherwise $r^{\prime}<r(A)$, so $1-2 r^{\prime}-\sigma>0$.

Let $\varepsilon \in\left(0, r^{\prime}\right)$. Take $x \in X(x \notin A)$ such that $r(A, x)<1-r^{\prime}-\sigma+\varepsilon$ and $y \in A$ such that $r^{\prime}(A, y)>r^{\prime}-\varepsilon$, so $r(A, y) \leq \delta(A)-r^{\prime}(A, y)<1-r^{\prime}+\varepsilon$. Along the segment joining $x$ and $y$ take $z \in \partial A, z=t x+(1-t) y$.

Note that $\|z-y\|=t\|x-y\|$ and $\|x-y\|+r^{\prime}(A, y) \leq r(A, x)<1-r^{\prime}-\sigma+\varepsilon$, so $t=\frac{\|z-y\|}{\|x-y\|}>\frac{r^{\prime}-\varepsilon}{1-r^{\prime}-\sigma+\varepsilon-\left(r^{\prime}-\varepsilon\right)}$.

By the convexity of $r(A,$.$) , we have:$

$r(A, z) \leq t r(A, x)+(1-t) r(A, y)<t\left(1-r^{\prime}-\sigma+\varepsilon\right)+(1-t)\left(1-r^{\prime}+\varepsilon\right)=$ $1-r^{\prime}+\varepsilon-t \sigma$.

So $1-r^{\prime}=r(A, A) \leq r(A, z)<1-r^{\prime}+\varepsilon-t \sigma$, which implies $\varepsilon-t \sigma>0$.

But $\varepsilon-t \sigma<\varepsilon-\sigma \frac{r^{\prime}-\varepsilon}{1-r^{\prime}-\sigma+\varepsilon-\left(r^{\prime}-\varepsilon\right)}$, and for $\varepsilon \rightarrow 0$ the right term has a negative limit.

So we have an absurdity, proving that $r(A)=1-r^{\prime}$, and then (5).

In general, also a ball (in particular, a complete set) can contain long segments on its boundary: think at a ball in $R^{2}$ with the max norm.

According to [6, Example 4], a set can satisfy (5) and contain segments on the boundary, also when $X$ is the Euclidean plane. We shall prove that the same cannot happen if $A$ satisfies (CR), so extending [13, Lemma 2].

Recall that a space $X$ is uniformly convex, (UC) for short, if and only if given $\alpha>0$ and $\varepsilon>0$, there exists $\sigma=\sigma(\varepsilon, \alpha)>0$ such that:

$$
\|x-a\| \leq \alpha,\|y-a\| \leq \alpha,\|y-x\| \geq \varepsilon \text { imply }\left\|\frac{x+y}{2}-a\right\| \leq \alpha-\sigma .
$$

Proposition 2.4. Let $X$ be a $(U C)$ space. If $A \subset X$ satisfies $(C R)$ (in particular, if $A$ is complete), then it cannot contain segments on its boundary.

Proof. Assume that the segment $[x, y]$ is contained in the boundary of a set $A$ satisfying (CR). Then, if $X$ is (UC), from $\|x-a\| \leq \delta(A)$ and $\|y-a\| \leq \delta(A)$ we would obtain $\left\|\frac{x+y}{2}-a\right\| \leq \delta(A)-\sigma$ for every $a \in A$ and some $\sigma>0$, against (CR). This contradiction proves the proposition. 
We recall the following well known result (Vlasov property).

Proposition 2.5. If $X^{*}$ is strictly convex, then the unbounded union of an increasing sequence of open balls is either an open halfspace, or the whole space.

Next result is similar to [17, Proposition 3.4].

Theorem 2.6. Let $X$ be reflexive and smooth (so $X^{*}$ is strictly convex). Then $(C W)$ is equivalent to $(C D)$.

Proof. As said at the beginning of this section, it is known that $(C W) \Rightarrow(C D)$.

Now let $X$ be reflexive and smooth. Assume that there exists a set $A$ satisfying (CD) but not $(\mathrm{CW})$. There exists $f$ in the unit ball of $X^{*}$ such that:

$\sup f(A)-\inf f(A)=\delta(A)-\sigma$ for some $\sigma>0$.

After a translation, we can assume that $0=\inf f(A)=f(O) ; \sup f(A)=$ $\delta(A)-\sigma$. Take $x$ orthogonal to the hyperplane $P=f^{-1}(0)$, from the side where the values of $f$ are negative $(-f(x)=1=\|x\|=\operatorname{distance}(x, P))$. According to $(\mathrm{CD})$, we have $r(A, n x)=\|n x\|+\delta(A)$. Denoting by $S(x, r)$ the open ball centered at $x$, with radius $r$, consider $\bigcup_{n \in N} S\left(n x,\|n x\|+\delta(A)-\frac{\sigma}{2}\right)$ : according to Proposition 2.5, this is an open halfspace containing $\{y \in X: f(y) \leq \delta(A)-\sigma\}$, thus $A$. Since $A$ is weakly compact, there exists $n_{o}$ such that $A \subset S\left(n_{0} x, n_{0} x+\right.$ $\left.\delta(A)-\frac{\sigma}{2}\right)$, so $r\left(A, n_{o} x\right) \leq\left\|n_{o} x\right\|+\delta(A)-\frac{\sigma}{2}$ : this contradiction proves the result.

\section{GOING TO BALLS}

We shall discuss now implications of the type: a (DM) set satisfying some other condition is a ball; we shall slightly improve the known results. Conditions of this type appear in the literature: see for example [3, Corollary 3.4], which generalizes [19, Lemma 1.8]: we shall generalize it below (see Remark 3.2). Another result is given in [17, Proposition 2.1]: this will be generalized by Corollary 3.3.

Other papers deal with this implication in Euclidean spaces or in Minkowski spaces: see $[1,5,12,16]$; see also [18, Theorem 4 and related comments].

Recall the following chain of inequalities for a set $D$ :

$2 r^{\prime}(D) \leq r(D)+r^{\prime}(D) \leq r(D, D)+r^{\prime}(D) \leq \delta(D) \leq 2 r(D) \leq 2 r(D, D)$.

The condition $r(D)=r^{\prime}(D)$ implies $r^{\prime}(D)=\delta(D) / 2$, and conversely.

Also: $r(D)=r^{\prime}(D)$ implies $r(D, D)=r^{\prime}(D)$; in turn this implies $r(D, D)=$ $r(D)$, and then $r(D)=r^{\prime}(D)$.

So we have:

$\left(B_{1}\right) \quad r(D)=r^{\prime}(D) \Longleftrightarrow r^{\prime}(D)=\frac{\delta(D)}{2} \Longleftrightarrow r(D, D)=r^{\prime}(D)$.

Thus each of the above equalities implies

$\left(B_{2}\right) \quad r(D, D)=\frac{\delta(D)}{2}$

which in turn implies 
$\left(B_{3}\right) \quad r(D)=\frac{\delta(D)}{2}$.

The converse implications do not hold; for $\left(B_{2}\right) \nRightarrow\left(B_{1}\right)$, think, in the Euclidean plane, at an ellipsis (this is also a symmetric set); for $\left(B_{3}\right) \nRightarrow\left(B_{2}\right)$, see Example 5.1 below. In fact, $\left(B_{2}\right)$ is equivalent to $\left(B_{3}\right)$ together with $r(D)=r(D, D)$ (the last condition is always satisfied by (DM) sets).

Some facts concerning sets satisfying $\left(B_{3}\right)$, in Minkowski spaces, are proved in [14, Section 4]. Note that there are spaces where all sets satisfy (3): see [9]; see also $[4,10]$.

We are ready to prove a simple result.

Theorem 3.1. A set $D$ is a ball if (and only if) it satisfies one of the equalities in $\left(B_{1}\right)$, so all them.

Proof. Let $D$ satisfy $\left(B_{1}\right)$. It is not a restriction to assume that $r(D)=r^{\prime}(D)=$ $1 ; \delta(D)=2$.

We prove first that $D$ has an incenter: namely, a point $c^{\prime}$ such that $r^{\prime}\left(D, c^{\prime}\right)=$ $r^{\prime}(D)$.

For every $n \in N$ there exists $x_{n}$ such that $B\left(x_{n}, 1-\frac{1}{n}\right) \subset D$. The sequence $\left(x_{n}\right)$ is Cauchy: in fact, we have $1-\frac{1}{n}+\left\|x_{n}-x_{m}\right\|+1-\frac{1}{m} \leq \delta(D)=2$, so $\left\|x_{n}-x_{m}\right\| \leq \frac{1}{n}+\frac{1}{m}$. Now let $x$ be the limit of $\left(x_{n}\right)$ : if $\|x-y\|=1-\varepsilon(\varepsilon>0)$, then we have $\left\|y-x_{n}\right\| \leq 1-\varepsilon+\left\|x-x_{n}\right\|$. Now let $n$ be large enough so that $\left\|x-x_{n}\right\|+\frac{1}{n}<\varepsilon$. Then $\left\|y-x_{n}\right\| \leq\|y-x\|+\left\|x-x_{n}\right\|<1-\frac{1}{n}$ for $n$ large enough, thus $y \in D$ : this shows that $B(x, 1-\varepsilon) \subset D$ for every $\varepsilon>0$, so $B(x, 1) \subset D$ (which is closed). Therefore $r^{\prime}(D, x)=1$, and then $x$ is an incenter of $D$.

But $B(x, 1) \neq D$ would imply the existence of $a \in D$ such that $\|x-a\|>1$, against $\delta(D)=2$. This proves that $D=B(x, 1)$.

Remark 3.2. Let $D$ be a set. It is clear that $(5)$ together with $\left(B_{3}\right)$ is equivalent to $(4)$ together with $\left(B_{2}\right)$, and these two conditions imply $\left(B_{1}\right)$; so (by Theorem 3.1) also that $D$ is a ball. This fact slightly improves [3, Corollary 3.4]. Compare with Remark 4.6.

We can prove the following result, which slightly improves [17, first part of Proposition 2.1].

Corollary 3.3. Let the following conditions hold: D satisfies (4) and is symmetric; then the set $D$ is a ball.

Proof. We may assume that $D$ is symmetric with respect to $O \quad($ so $O \in D)$ : otherwise we consider a translation carrying the center of symmetry to $O$.

Set $r=r(D, O)$. We note first that $\delta(D)=2 r(D)$ : in fact, for any $\sigma>0$, there is a point $x \in D$ such that $\|x\|>r-\sigma$, then $-x \in D$ thus $\|x-(-x)\|>2 r-2 \sigma$; so $2 r \leq \delta(D) \leq 2 r(D) \leq 2 r(D, D) \leq 2 r(D, O)=2 r$, and then the equality. So $O$ is a center of $D$ and $\left(B_{2}\right)$ holds; thus the conclusion according to the preceding remark.

Next proposition generalizes [17, second part of Proposition 2.1]. 
Proposition 3.4. If a set $A$ satisfies (4) and is compact, then the interior of $A$ is nonempty (so the underlying space $X$ must be finite-dimensional).

Proof. Let $A$ be compact; this implies (see for example [11, p. 39]) that there is a point $a \in A$ such that $r(A, a)<\delta(A)$, so $r(A, A)<\delta(A)$, and then (by (4)) $r^{\prime}(A)>0$. This cannot be true if $\operatorname{dim}(X)=\infty$.

Remark 3.5. The preceding result says that when $\operatorname{dim}(X)=\infty$, a set satisfying (4) (in particular, a (DM) set) cannot be compact. So, for example, completing a compact set requires the addition of a "rather big" set.

\section{Completions And Balls}

Consider the following condition for a set $D$ :

$\left(B_{4}\right)$ one of the completions of $D$ is a ball.

Next result shows the following fact: a set satisfies $\left(B_{4}\right)$ if and only if it satisfies $\left(B_{3}\right)$ and has centers.

Theorem 4.1. If a set $D$ satisfies $\left(B_{4}\right)$, then it satisfies $\left(B_{3}\right)$; moreover, the center(s) of the ball(s) that are completions of $D$, are centers of $D$.

Conversely, if $D$ satisfies $r(D)=\delta(D) / 2$ and $c$ is a center of $D$, then the ball $B(c, r(D))$ is a completion of $D$.

Proof. Let $D$ satisfy $\left(B_{4}\right)$ and let the ball $B=B(c, r)$ be a completion of $D$. Then we have:

$\delta(B)=\delta(D) ; r(D) \leq r=\delta(B) / 2=\delta(D) / 2$, so $r=r(D)=\delta(D) / 2$, which is $\left(B_{3}\right)$. Also, $r(D, c)=r(D)$, so we have the first part of the thesis.

Conversely, let $D$ satisfy $\left(B_{3}\right)$ and let $c$ be a center of $D$; then $B(c, r(D))$, which is clearly a complete set with diameter $\delta(D)$, is a completion of $D$ : thus $\left(B_{4}\right)$ holds.

The following fact was proved in [3, Theorem 3.5]:

Proposition 4.2. Let $D \subset B(x, r)$ (for some $x \in X$ and $r \in R$ ); then, if $r \leq$ $\delta(D), D$ has a completion contained in $B(x, r)$.

According to the above result, the infimum of the radii of completions of $D$ is $r(D)$. In particular, if $D$ has a unique completion, then the radius of its completion is $r(D)$.

Proposition 4.3. Let $D$ satisfy $\left(B_{3}\right)$; if $D$ has a completion $D_{m}$ with $r\left(D_{m}\right)=$ $r(D)$, then $D_{m}$ is a ball (and $D$ has a center).

In particular, this happens if $D$ has a unique completion (and in this case $D$ has a unique center).

Proof. We have: $r\left(D_{m}\right)=r(D)=\delta(D) / 2=\delta\left(D_{m}\right) / 2$, so $D_{m}$ satisfies $\left(B_{3}\right)$. But a complete set satisfying $\left(B_{3}\right)$ is a ball (see Remark 3.2 ); also, its center is a center of $D$ by Theorem 4.1. 
Remark 4.4. We discuss now the different possibilities concerning completions of a set $D$ satisfying $\left(B_{3}\right)$.

i) No completion of $D$ is a ball, or - equivalently - (see Theorem 4.1) $D$ has no center. Then according to Proposition 4.3, $D$ has more completions (not balls), and all of them have radius $>r(D)$.

So, if the completion of $D$ is unique (like in Example 5.1), it is necessarily a ball.

ii) $D$ has a completion which is a ball: then, according to Theorem 4.1, the centers of balls which are completions coincide with the centers of $D$, so the radius of these balls is $r(D)$; completions of this type can be unique (think, for example at a segment in the Euclidean plane), or multiple (think at a segment in the plane with the max norm). Uniqueness of such completions certainly holds if the space $X$ is such that centers of sets are unique (like Hilbert spaces). But $D$ can have also other completions, not balls, with radius $>r(D)$ (according to Proposition 4.3): see also [3, Example 3.3]. As Example 5.3 shows, we can have different completions of both types.

Note that in general $\left(B_{3}\right)$ does not imply the existence of centers for $D$ (see [6, Example 2]): so $\left(B_{3}\right) \nRightarrow\left(B_{4}\right)$.

Example 5.4 will show that $\left(B_{2}\right)$ does not imply existence of centers; thus $\left(B_{2}\right) \nRightarrow\left(B_{4}\right)$.

Remark 4.5. Let $D$ satisfy $\left(B_{3}\right)$. Take $\tilde{D} \subset D ; \delta(\tilde{D})=\delta(D)$ : then also $\tilde{D}$ satisfies $\left(B_{3}\right)$ : in fact $r(\tilde{D}) \leq r(D)=\delta(D) / 2=\delta(\tilde{D}) / 2$. Then $\tilde{D}$ can have other completions. Moreover it is possible that $D$ has no center and $\tilde{D}$ has.

Remark 4.6. We have $\left(B_{4}\right) \nRightarrow\left(B_{2}\right)$; see Example 5.1, where the center of the ball which is a completion of $D$ does not belong to $D$.

The same example shows that for a set, $\left(B_{4}\right)$, together with the condition $(\mathrm{CR})$, does not imply that it is a ball.

We resume part of our discussion with the following scheme; the only true implications are those indicated below.

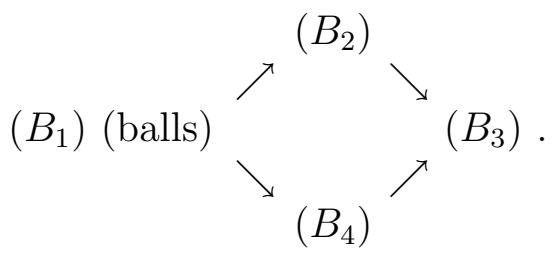

If $X$ is finite-dimensional, or it is a Hilbert space, then $\left(B_{4}\right) \Leftrightarrow\left(B_{3}\right)$; if $X$ is two-dimensional, or it is a Hilbert space, then $\left(B_{2}\right) \Leftrightarrow\left(B_{3}\right)$; Example 5.5 will show that the last equivalence is not true if $X$ is three-dimensional. 


\section{EXAMPLES}

The first two examples are related to the condition $(\mathrm{CR}+)$. The first one shows that $(\mathrm{CR}) \nRightarrow(\mathrm{CR}+)$; the second that $(\mathrm{CR}+) \nRightarrow$ completeness.

Example 5.1. Let $D=\{f \in C[0,1]: f(0)=0 ; 0 \leq f(x) \leq 1\}$; its unique completion is $B=B(\bar{f}, 1 / 2)$, where $\bar{f}$ is the constant function $1 / 2$ (we have $r(D)=r(B)=r(B, B)=1 / 2 ; r(D, D)=1=\delta(D)=\delta(B))$.

Example 5.2. Consider the space $c_{o}$ of all sequences converging to 0, with the $\max$ norm. Let $D=\left\{x=\left(x_{1}, x_{2}, \cdots, x_{n}, \cdots\right): x_{1}=0 ; 0 \leq x_{i} \leq 1\right.$ for $i \geq$ 2\} $(\delta(D)=1)$. The set $D$ has different completions. If $D^{\prime}$ denotes the union of its completions, we have: $\delta\left(D^{\prime}\right)=2 ; r(D)=r\left(D^{\prime}\right)=r(D, D)=1$.

Next example is related to the second part of Remark 4.4 (set with different completions).

Example 5.3. Let $D=\{f \in C[-1,1]: f(x)=0$ for $-1 \leq x \leq 0 ; 0 \leq f(x) \leq 1$ for $0 \leq x \leq 1\}$; one of its completions is $D_{m}=B(\bar{f}, 1 / 2)$, where $\bar{f}$ is the constant function $1 / 2 \quad$ (we have $r(D)=r\left(D_{m}\right)=r\left(D_{m}, D_{m}\right)=1 / 2 ; \quad r(D, D)=1=$ $\left.\delta(D)=\delta\left(D_{m}\right)\right)$.

Example 5.4, a development of [2, Example 5.2], shows that also a set satisfying $\left(B_{2}\right)$, can have no center.

Example 5.4. Consider $c_{0}$, the usual space of real sequences $\left(x_{1}, x_{2}, \ldots, x_{n}, \ldots\right)$ with the sup norm. Set $X=\left\{x \in c_{0}: \sum_{n=1}^{\infty} f_{n} x_{n}=0\right\}$ where $f_{n}=1$ for $n=1,2,3,4$ and $f_{n}=\frac{1}{2^{n-4}}$ for $n \geq 5$. Denote by $e_{i}$ the elements of the natural basis of $c_{0}$. Let $A=\{a, b, c\}$ where $a=e_{1}-e_{4} ; b=e_{2}-\frac{1}{2} e_{4}-e_{5} ; c=e_{3}-\frac{1}{2} e_{4}-e_{5}$. We have: $\|a-b\|=\|a-c\|=\|b-c\|=1$. Now set, for $\varepsilon>0: x^{\varepsilon}=$ $\left(\frac{1}{2}-\varepsilon, \frac{1}{2}-\varepsilon, \frac{1}{2}-\varepsilon,-1-\varepsilon, \frac{-1}{2}-\varepsilon, \ldots, \frac{-1}{2}-\varepsilon, x_{n+5}, \ldots\right)\left(x=\frac{-1}{2}-\varepsilon n\right.$ times) where $x_{k}=0$ for $k \geq n+5$. If $\varepsilon<\frac{1}{2}$, we have $\left\|x^{\varepsilon}-a\right\|=\left\|x^{\varepsilon}-b\right\|=\left\|x^{\varepsilon}-c\right\|=\frac{1}{2}+\varepsilon$; moreover, by taking $\varepsilon=\frac{1}{2\left(52^{n}-1\right)}$ we also have $f\left(x^{\varepsilon}\right)=0$. For $n$ large, $\varepsilon$ becomes as small as we wish.

As shown in [2, Example 5.2], the set $A$ satisfies: $\delta(A)=1 ; r(A)=1 / 2$ (more precisely, $\left.r\left(A, x^{\varepsilon}\right)=\frac{1}{2}+\varepsilon\right) ; A$ has no center.

Now let $\tilde{A}$ be the closed convex hull of $A \cup\left\{x^{\varepsilon}: \varepsilon=\frac{1}{2\left(52^{n}-1\right)} ; n \in N\right\}$. Again, $\delta(\tilde{A})=1 ; r(\tilde{A})=1 / 2$, and also $r(\tilde{A}, \tilde{A})=1 / 2$ : in fact, $r\left(\tilde{A}, x^{\varepsilon}\right)=1 / 2+\varepsilon$ and $\varepsilon \rightarrow 0$ for $n \rightarrow \infty$.

So, of course, $\tilde{A}$ has no center (and no self center).

Next example shows that in general $\left(B_{2}\right)$ and $\left(B_{4}\right)$ are not equivalent in spaces of dimension larger that 2 .

Example 5.5. Let $X=\ell_{1}^{3}\left(X=R^{3}\right.$ with the sum norm). Take

$D=\{(1,0,0),(0,1,0),(0,0,1)\}$. Then $D \subset B(O, 1)$, so $r(D)=1 ; \delta(D)=2$. But $r(D, D)=4 / 3>\delta(D) / 2$. The origin is the unique center of $D$. 
The set $D$ has other completions; for example, set $\tilde{D}=D \cup\left\{\left(\frac{1}{2}, \frac{1}{2}, \frac{1}{2}\right)\right\}$. We have: $\delta(\tilde{D})=2 ; B(O, 1)$ does not contain $\tilde{D}$; so $\tilde{D}$ has other completions, which are also completions of $D$.

\section{REFERENCES}

1. G. Averkov, On boundary arcs joining antipodal points of a planar convex body, Beiträge Alg. Geom. 47 (2006), no. 2, 489-503.

2. M. Baronti, E. Casini and P.L. Papini, Equilateral sets and their central points, Rend. Mat. Appl.(7) 13 (1993), no. 1, 133-148.

3. M. Baronti and P.L. Papini, Diameters, centers and diametrically maximal sets, IVth Italian Conference on Integral Geometry, Geometric Probability Theory and Convex Bodies (Bari, 1994). Rend. Circ. Mat. Palermo (2) Suppl. No. 38 (1995), 11-24.

4. J.M. Bayod and M.C. Masa, Chebyshev coefficients for $L^{1}$-preduals and for spaces with the extension property, Publ. Mat. 34 (1990), no. 2, 341-147.

5. K. Böröczky, Jr., Rectangular convexity of convex domains of constant width, Geom. Dedicata 34 (1990), 13-18.

6. L. Caspani and P.L. Papini, Complete sets, radii, and inner radii, Beiträge Alg. Geom. 52 (2011), 163-170.

7. L. Caspani and P.L. Papini, On constant width sets in Hilbert spaces and around, J. Convex Anal. (to appear).

8. G.D. Chakerian and H. Groemer, Convex bodies of constant width, in Convexity and its applications, (P. Gruber and J. Wills Eds.), Birkhäuser, Basel, 1983, 49-96.

9. W.J. Davis, A characterization of $\mathcal{P}_{1}$ spaces, J. Approx. Theory 21 (1977), 315-318.

10. Y. Duan and B.-L. Lin, Characterizations of $L^{1}$-predual spaces by centerable subsets, Comment. Math. Univ. Carolin. 48 (2007), 239-243.

11. K. Goebel and W.A. Kirk, Topics in metric fixed point theory, Cambrigde Univ. Press, Cambridge 1990.

12. R. Howard and D. Hug, Nakajima's problem: convex bodies of constant width and constant brightness, Mathematika 54 (2007), 15-24.

13. H. Jin and Q. Guo, Asymmetry of convex bodies of constant width, Discrete Comput. Geom. 47 (2012), 415-423.

14. P. Martín, H. Martini and M. Spirova, Chebyshev sets and ball operators, J. Convex Anal. 21 (2014), 601-618.

15. H. Martini and K.J. Swanepoel, The geometry of Minkowski spaces - a survey. II, Expo. Math. 22 (2004), 93-144.

16. L. Montejano, A characterization of the Euclidean ball in terms of concurrent sections of constant width, Geom. Dedicata 37 (1991), 307-316.

17. J.P. Moreno, P.L. Papini and R.R. Phelps, New families of convex sets related to diametral maximality, J. Convex. Anal. 13 (2006), 823-837.

18. J.P. Moreno and R. Schneider, Diametrically complete sets in Minkowski spaces, Israel J. Math. 191 (2012), 701-720.

19. R. Payá and A. Rodríguez-Palacios, Banach spaces which are semi-L-summands in their biduals, Math. Ann. 289 (1991), 529-542.

20. E.S. Polovinkin, On the construction of bodies of constant width containing a given set, Proc. Steklov Inst. Math. (2010), suppl.2, S247-S257.

Via Martucci, 19, 40136 Bologna, Italy.

E-mail address: plpapini@libero.it 